\title{
On resonance search in dilepton events at the LHC
}

\author{
M. V. Chizhov ${ }^{1,2}$, V. A. Bednyakov ${ }^{1}$, J. A. Budagov ${ }^{1}$ \\ ${ }^{1}$ Dzhelepov Laboratory of Nuclear Problems, \\ Joint Institute for Nuclear Research, 141980, Dubna, Russia \\ ${ }^{2}$ Centre for Space Research and Technologies, \\ Faculty of Physics, Sofia University, 1164 Sofia, Bulgaria
}

\begin{abstract}
The main distribution for a bump search is the dilepton invariant mass distribution with appropriated cut on an absolute value of pseudorapidity difference $\Delta \eta \equiv\left|\eta_{1}-\eta_{2}\right|$ between the two leptons. The background from the Standard Model Drell-Yan process contributes mainly to the central pseudorapidity region $\Delta \eta \simeq 0$. By contrast, the excited bosons lead to a peak at $\Delta \eta \simeq 1.76$. We show that this property allows to enhance the significance of their bump search by means of new cut optimization. Nevertheless, in order to confirm an observation of the bump and reveal the resonance nature other angular distributions should be used in addition.
\end{abstract}

PACS numbers: 12.60.-i, 13.85.-t, 14.80.-j

\section{INTRODUCTION}

The golden channel of resonances search at the hadron colliders is the lepton one with electrons or muons in a final state. Free from huge QCD background it opens clean way of the resonance identification. Depending on the resonance electric charge there are two possible signatures of the resonances search through their decay into a charged lepton and a neutrino with missing transverse momentum or into a lepton pair (Drell-Yan production process). The latter has the very clean signature and almost totally reconstructible kinematics ${ }^{1}$, which allows investigation of the production and the decay of the new resonances.

In this letter we will consider the case of the resonance production of new bosons up to spin 2 and their detection in the Drell-Yan process using the first CERN LHC data. We will concentrate mainly on the case of excited bosons, which have unique angular distribution. The simple model of the excited bosons was proposed in [1]. Roughly speaking it leads to a signal, $s$, of the two orders of magnitude higher than the Standard Model (SM) background, $b$. Therefore, as for the other benchmark models, the direct exclusion limits on mass of $1.152 \mathrm{TeV}$ and on cross section times branching ratio $\sigma B\left(Z^{*} \rightarrow \ell^{+} \ell^{-}\right)<89 \mathrm{fb}$ have been obtained for the first time, based on 2010 ATLAS data [2].

In general case the coupling constants of the new bosons with quarks and leptons could be weaker and their masses could be lighter than the obtained limit. In this case the signal significance, $s / \sqrt{b}$, will decrease, which lead to more complicated situation in the resonance search. The main background comes from the inclusive Drell-Yan process $p p \rightarrow \gamma / Z \rightarrow \ell^{+} \ell^{-}$with $\gamma / Z$ exchanges and is practically unremovable. Therefore, instead to wait for more data one can involve angular dis-

\footnotetext{
${ }^{1}$ Up to small individual transverse momenta of the quarks.
}

tributions into analysis [3] as well.

We will use unique properties of angular distributions of the excited bosons in order to increase their signal sensitivity in the present data. In the next section we will consider a model independent signal distributions of new physics from resonances up to spin 2. Present analysis is based on our study of dijet mass angular distributions [4], which has been shown to play important role in disentangling of the resonance properties and revealing the unique signature of the excited bosons.

\section{A UNIQUE SIGNAL OF EXCITED BOSONS}

The distribution of final state lepton over the polar scattering angle $\theta$, being angle between the axis of the lepton pair and the beam direction in the dilepton rest frame, is directly sensitive to the dynamics of the underlying process, where the spins of the resonance and of the initial and final states uniquely define the angular distribution.

In the following we will consider a distribution on the absolute value of the dilepton pseudorapidity difference, which is related to the angle $\theta$ by $\Delta \eta \equiv\left|\eta_{1}-\eta_{2}\right|=\ln [(1+$ $|\cos \theta|) /(1-|\cos \theta|)] \geq 0$. It has been shown [5] that the excited boson distribution on $\Delta \eta$ drastically differ from the SM and other exotic models. So, while the SM processes are dominated by $\gamma / Z$ exchanges, which lead to well-known $1+\cos ^{2} \theta$ distribution ${ }^{2}$, exotic physics processes can deviate from this distribution.

Let us consider different possibilities for the spin of a resonance and its possible interactions with quarks and leptons. The simplest case of the resonance production of a (pseudo)scalar particle $h$ with spin 0 in $s$-channel leads to a uniform decay distribution on the scattering

\footnotetext{
2 The choice of the variables, which depend on the absolute value of $\cos \theta$, cancels out the apparent dependence on $\cos \theta$.
} 
angle

$$
\frac{\mathrm{d} \Gamma_{0}\left(h \rightarrow \ell^{+} \ell^{-}\right)}{\mathrm{d} \cos \theta} \propto\left|d_{00}^{0}\right|^{2} \sim 1
$$

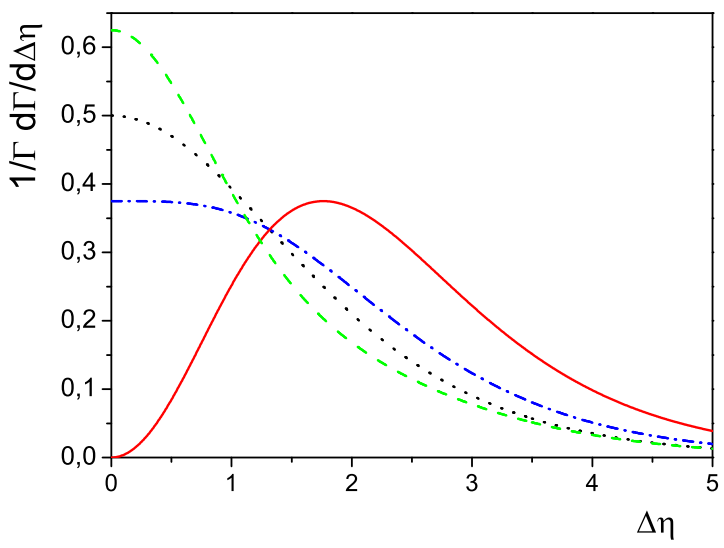

FIG. 1: The normalized angular lepton distributions as functions of $\Delta \eta$ for the scalar, spin-1 bosons with the minimal couplings, the excited bosons and spin-2 resonances are shown with the dotted, dash-dotted, solid and dashed curves, respectively.

\section{Variable transformation}

$$
\frac{\mathrm{d} \Gamma}{\mathrm{d} \Delta \eta}=\frac{\mathrm{d} \cos \theta}{\mathrm{d} \Delta \eta} \frac{\mathrm{d} \Gamma}{\mathrm{d} \cos \theta},
$$

in the uniform distribution (1) from $\cos \theta$ to $\Delta \eta$

$$
\frac{1}{\Gamma_{0}} \frac{\mathrm{d} \Gamma_{0}}{\mathrm{~d} \Delta \eta}=\frac{2 \mathrm{e}^{\Delta \eta}}{\left(\mathrm{e}^{\Delta \eta}+1\right)^{2}}
$$

leads to kinematical peak at $\Delta \eta=0$ (the dotted curve in Fig. 1), which corresponds to the polar angle $\theta=90^{\circ}$. The result coincides with common opinion, that an expected signal of new physics should be in perpendicular direction to the beam.

According to [4, 6] there are only two different possibilities for the spin-1 resonances to interact with light fermions. All known gauge bosons have minimal interactions with the light fermions

$$
\mathcal{L}_{Z^{\prime}}=\sum_{f}\left(g_{L L}^{f} \overline{\psi_{L}^{f}} \gamma^{\mu} \psi_{L}^{f}+g_{R R}^{f} \overline{\psi_{R}^{f}} \gamma^{\mu} \psi_{R}^{f}\right) Z_{\mu}^{\prime},
$$

which preserve the fermion chiralities and possess maximal helicities $\lambda= \pm 1$. It is assumed that the hypothetical $Z^{\prime}$ bosons have also similar couplings. At a symmetric $p p$ collider, like the LHC, such interactions lead to the specific symmetric angular distribution of the resonance decay products

$$
\frac{\mathrm{d} \Gamma_{1}\left(Z^{\prime} \rightarrow \ell^{+} \ell^{-}\right)}{\mathrm{d} \cos \theta} \propto\left|d_{11}^{1}\right|^{2}+\left|d_{-11}^{1}\right|^{2} \sim 1+\cos ^{2} \theta .
$$

Similar to the uniform distribution, eq. (5) also leads to kinematical peaks at $\Delta \eta=0$ (the dash-dotted curve in Fig. 1):

$$
\frac{1}{\Gamma_{1}^{\prime}} \frac{\mathrm{d} \Gamma_{1}^{\prime}}{\mathrm{d} \Delta \eta}=\frac{3 \mathrm{e}^{\Delta \eta}\left(\mathrm{e}^{2 \Delta \eta}+1\right)}{\left(\mathrm{e}^{\Delta \eta}+1\right)^{4}} .
$$

Another possibility is the resonance production and decay of new longitudinal spin-1 $Z^{*}$ bosons with helicity $\lambda=0$. The new gauge bosons with such properties arise in many extensions [6] of the SM, which solve the Hierarchy Problem.

While the $Z^{\prime}$ bosons with helicities $\lambda= \pm 1$ are produced in left(right)-handed quark and right(left)-handed antiquark fusion, the longitudinal $Z^{*}$ bosons are produced through the anomalous chiral couplings with the ordinary light fermions

$$
\mathcal{L}_{Z^{*}}=\sum_{f} \frac{g_{R L}^{f}}{M} \overline{\psi_{R}^{f}} \sigma^{\mu \nu} \psi_{L}^{f} \partial_{[\mu} Z_{\nu]}^{*}+\text { h.c. }
$$

in left-handed or right-handed quark-antiquark fusion [1]. The anomalous interactions (7) are generated on the level of the quantum loop corrections and can be considered as effective interactions. The $Z^{*}$ resonances are some types of "excited" states as far as the only orbital angular momentum with $L=1$ contributes to the total angular moment, while the total spin of the system is zero. This property manifests itself in their derivative couplings to fermions and a different chiral structure of the interactions in contrast to the minimal gauge interactions (4).

The anomalous couplings lead to a different angular distribution

$$
\frac{\mathrm{d} \Gamma_{1}\left(Z^{*} \rightarrow \ell^{+} \ell^{-}\right)}{\mathrm{d} \cos \theta} \propto\left|d_{00}^{1}\right|^{2} \sim \cos ^{2} \theta
$$

than the previously considered ones. A striking feature of the distribution is the forbidden decay direction perpendicular to the beam. It leads to a profound dip at $\cos \theta=0$ [1]. The same dip presents also at $\Delta \eta=0[\underline{5}]$ (the solid curve in Fig. 11):

$$
\frac{1}{\Gamma_{1}^{*}} \frac{\mathrm{d} \Gamma_{1}^{*}}{\mathrm{~d} \Delta \eta}=\frac{6 \mathrm{e}^{\Delta \eta}\left(\mathrm{e}^{\Delta \eta}-1\right)^{2}}{\left(\mathrm{e}^{\Delta \eta}+1\right)^{4}} .
$$

It can be seen from Fig. 1 that the excited bosons have unique signature in the angular distribution. They manifest themselves through the absolute minimum at $\Delta \eta=0$ and absolute maximum at $\Delta \eta=\ln (3+\sqrt{8}) \approx 1.76$. The latter corresponds to the polar angle $\theta=45^{\circ}$ and a little bit contradicts to the common opinion about an expected signal from new physics.

The spin-2 resonances, like Kaluza-Klein excitations, lead to the following decay distributions depending on initial parton configurations

$$
\frac{\mathrm{d} \Gamma_{g g}\left(G^{*} \rightarrow \ell^{+} \ell^{-}\right)}{\mathrm{d} \cos \theta} \propto\left|d_{2,1}^{2}\right|^{2}+\left|d_{2,-1}^{2}\right|^{2} \sim 1-\cos ^{4} \theta
$$


and

$$
\begin{aligned}
\frac{\mathrm{d} \Gamma_{q \bar{q}}\left(G^{*} \rightarrow \ell^{+} \ell^{-}\right)}{\mathrm{d} \cos \theta} & \propto\left|d_{1,1}^{2}\right|^{2}+\left|d_{1,-1}^{2}\right|^{2} \\
& \sim 1-3 \cos ^{2} \theta+4 \cos ^{4} \theta .
\end{aligned}
$$

Taking into account their corresponding weights, $3 / 5$ and $2 / 5$, for the inclusive process $p p \rightarrow G^{*} \rightarrow \ell^{+} \ell^{-}$[7] and using eq. (2) one can obtain the distribution on $\Delta \eta$ (the dashed curve in Fig. (1)

$$
\frac{1}{\Gamma_{2}} \frac{\mathrm{d} \Gamma_{2}}{\mathrm{~d} \Delta \eta}=\frac{2 \mathrm{e}^{\Delta \eta}\left(\mathrm{e}^{4 \Delta \eta}+18 \mathrm{e}^{2 \Delta \eta}+1\right)}{\left(\mathrm{e}^{\Delta \eta}+1\right)^{6}},
$$

which also peaks at $\Delta \eta=0$ as in the most exotic models.

\section{CUT OPTIMIZATION FOR EXCITED BOSONS}

From Fig. 1 one can see that all distributions besides of the excited bosons peak at $\Delta \eta=0$. Since the main background comes from the $\gamma / Z$ bosons, which have the minimal gauge couplings with quarks and lepton, they also populate the central pseudorapidity region. Therefore, it is difficult to disentangle new physics signal using this distribution unless in the case of the excited bosons. Applying the proper cut $\Delta \eta>a$ one can suppress unwanted background contribution and enhance the signal significance, $\mathcal{S}=s / \sqrt{b}$, choosing its maximum.

So, we can define the relative significance of $Z^{*}$ signal to $Z^{\prime}$-like background as the ratio of the definite integral from $a$ to infinity of the normalized signal distributions (9) to the square root of the SM background, dominated by distribution (6),

$$
\mathcal{S}(a)=\frac{\int_{a}^{\infty} \frac{1}{\Gamma_{1}^{*}} \frac{\mathrm{d} \Gamma_{1}^{*}}{\mathrm{~d} \Delta \eta} \mathrm{d} \Delta \eta}{\sqrt{\int_{a}^{\infty} \frac{1}{\Gamma_{1}^{\prime}} \frac{\mathrm{d} \Gamma_{1}^{\prime}}{\mathrm{d} \Delta \eta} \mathrm{d} \Delta \eta}}=\frac{1-\left(\frac{\mathrm{e}^{a}-1}{\mathrm{e}^{a}+1}\right)^{3}}{\sqrt{1-\frac{\mathrm{e}^{3 a}-1}{\left(\mathrm{e}^{a}+1\right)^{3}}}}
$$

The distribution reaches the maximum value around 1.14 at $a \approx 1.02$ (Fig. 22).

The corresponding cut at the maximum signal significance suppresses around $38 \%$ of the background and only
$10 \%$ of the signal. This leads to relative enhancement in sensitivity, which is equivalent to adding approximately $29 \%$ of data in comparison with usual sensitivity without any cuts.

\section{CONCLUSIONS}

In this paper we have presented $\Delta \eta$-distributions for all possible resonanses with spin up to 2 . On this basis we have proposed the novel optimization of angular distribution cut aimed at the most effective search for the new resonances with different angular distributions in dilepton events. In particular, this allows to enhance the significance of bump search for the excited bosons.

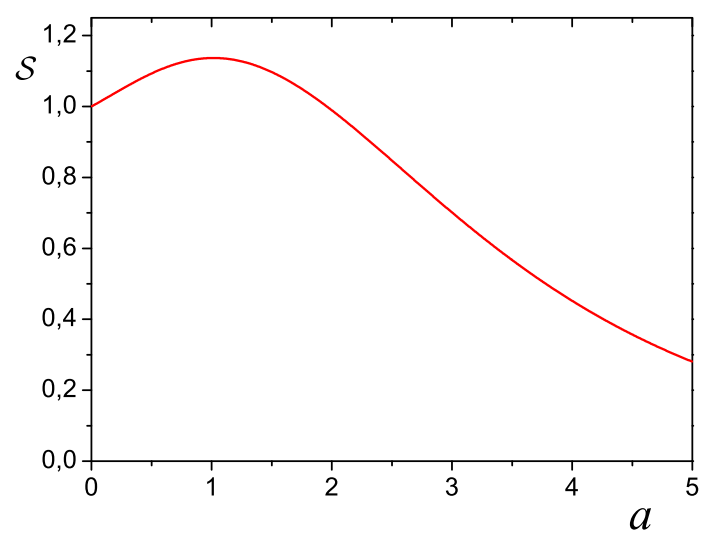

FIG. 2: The relative signal significance distribution of the excited bosons as functions of the cut parameter $a$ on $\Delta \eta$.

\section{Acknowledgements}

The work of M.V. Chizhov was partially supported by the grant of Plenipotentiary of the Republic of Bulgaria in JINR for 2011 year.
[1] M.V. Chizhov, V.A. Bednyakov and J.A. Budagov, Phys. Atom. Nucl. 71 (2008) 2096, arXiv:0801.4235 [hep-ph].

[2] The ATLAS Collaboration, Phys. Lett. B 700 (2011) 163, arXiv:1103.6218 [hep-ex].

[3] The CDF Collaboration, Phys. Rev. Lett. 96 (2006) 211801.

[4] M.V. Chizhov, V.A. Bednyakov and J.A. Budagov, Phys. Atom. Nucl. 75 (2012) 1, arXiv:1010.2648 [hep-ph];
arXiv:1106.4161 [hep-ph].

[5] M.V. Chizhov, V.A. Bednyakov and J.A. Budagov, Nuovo Cimento 33C (2010) 343, arXiv:1005.2728 [hep-ph].

[6] M.V. Chizhov and Gia Dvali, Phys. Lett. B 703 (2011) 593, arXiv:0908.0924 [hep-ph].

[7] J. Bijnens et al., Phys. Lett. B 503 (2001) 341. 